\title{
Comparative Distribution of Glutamate Transporters and Receptors in Relation to Afferent Innervation Density in the Mammalian Cochlea
}

\author{
David N. Furness and D. Maxwell Lawton \\ MacKay Institute of Communication and Neuroscience, School of Life Sciences, Keele University, Keele, Staffordshire, ST5 5BG, United Kingdom
}

The local expression of proteins involved in handling glutamate may be regulated by the number and activity of synapses in regions of glutamatergic innervation. The systematically varying innervation of inner hair cells (IHCs) of the cochlea provides a model to test this suggestion. IHCs are glutamatergic and form a single row along the cochlear spiral. Along this row the number of afferent fibers terminating on IHCs increases toward the base, reaching a peak and thereafter declining. The afferents are segregated so that higher spontaneous rate fibers terminate on the pillar-cell side of the IHC and lower rate fibers terminate on the modiolar side. Using immunofluorescence and postembedding immunogold labeling, we investigated the distributions of the glutamate-aspartate transporter (GLAST or excitatory amino acid transporter 1), vesicular glutamate transporter (VGLUT1), and the AMPA receptor glutamate receptor 4 (GluR4) along the spiral. Immunofluorescent labeling for GLAST in IHC supporting cells increased in intensity to a peak in the region of 6-9 mm from the apex. Immunogold labeling for GLAST was greater overall in these cells in the $10 \mathrm{~mm}$ region than in the $1 \mathrm{~mm}$ region and also on the pillar-cell side of the IHC compared with the modiolar side. Immunogold labeling for GluR4 was confined to synaptic sites, represented by puncta in immunofluorescence. The relative numbers of puncta changed with a gradient similar to that of GLAST labeling. VGLUT1 labeling occurred in IHCs but showed no clear cochleotopic gradient. These data suggest that both the density of innervation and the activity levels of glutamatergic synapses may be involved in modulating regional expression of GLAST.

Key words: glutamate; cochlea; inner hair cells; GLAST; GluR4; VGLUT1; EAAT1

\section{Introduction}

The mammalian cochlea provides a useful model for the study of factors that may modulate mechanisms of glutamate handling at glutamatergic synapses (Ottersen et al., 1998). In particular, the cochlea exhibits systematic variations in the number and activity of glutamatergic synapses between afferent fibers and the two types of hair cell. The inner hair cells (IHCs) are organized in a single longitudinal row between the apex and base of the cochlea, each innervated by unbranched radial afferent fibers from up to 20 separate spiral ganglion neurons, in total constituting $90-95 \%$ of all afferent fibers in the auditory nerve. The outer hair cells (OHCs) are present in three rows and are innervated by the remaining $5-10 \%$ of fibers, which are branched and contact several OHCs (Spoendlin, 1972).

A range of proteins associated with glutamate release and recycling are present in the vicinity of the hair cell-afferent nerve synapses. For example, the glutamate-aspartate transporter (GLAST) [or excitatory amino acid transporter 1 (EAAT1)] occurs in supporting cells around the hair cells (Furness and Lehre, 1997), and AMPA-receptor subunits glutamate receptor $2 / 3$

\footnotetext{
Received July 14, 2003; revised 0ct. 3, 2003; accepted 0ct. 3, 2003.

This work was supported by a grant from the Wellcome Trust to D.N.F. We thank Dr. A. Polwart for advice on statistics and L. Bilsborrow and K. Walker for technical assistance.

Correspondence should be addressed to Dr. D. N. Furness, MacKay Institute of Communication and Neuroscience, School of Life Sciences, Keele University, Keele, Staffordshire, ST5 5BG, UK. E-mail: d.n.furness@keele.ac.uk. Copyright $\odot 2003$ Society for Neuroscience ～0270-6474/03/2311296-09\$15.00/0
}

(GluR2/3) and GluR4 (Matsubara et al., 1996; Ottersen et al., 1998; Parks, 2000) and metabotropic glutamate receptors (Oestreicher et al., 2002) occur at the IHC synapses. In addition, hair cells contain phosphate-activated glutaminase, which is involved in the synthetic pathway for glutamate (Takumi et al., 1999), and mRNA for a vesicular glutamate transporter (VGLUT1) (Bellochio et al., 2000) has been extracted from cochlear hair-cell preparations (Cheatham et al., 2001).

The local amount of GLAST expression appears to correspond with the differences in hair-cell innervation. Immunolabeling for GLAST is greater in the supporting cells in the IHC region (Furness and Lehre, 1997; Hakuba et al., 2000; Rebillard et al., 2003) than in Deiters' cells supporting the OHCs (Furness et al., 2002). The amount of GLAST in Deiters' cells is also greater in the apical region (Furness et al., 2002), where OHC afferent innervation density is highest (Hashimoto and Kimura, 1988; Liberman et al., 1990). These observations lead us to suggest the hypothesis that GLAST expression is locally regulated by the amount of glutamatergic activity. This will in turn depend on the density and activity levels of the afferent innervation to the hair cells.

As is the case with OHCs, there is systematic variation in innervation associated with IHCs that provides an additional opportunity to test this hypothesis. Along the IHC row, the number of afferent terminals per IHC increases to a peak in the mid-basal turns, after which it decreases (Liberman et al., 1990). Moreover, fibers with higher spontaneous rates (SRs) of action potentials 
tend to terminate on the pillar-cell side of the IHC, whereas lowmedium SR fibers terminate on the modiolar side (Liberman, 1982). We therefore have investigated whether the distribution and relative expression of GLAST varies in relation to IHC afferent innervation density and activity in the guinea pig cochlea and have examined the distribution of GluR4 and VGLUT1 to determine whether there are comparable variations in these other glutamate-associated proteins.

\section{Materials and Methods}

Animals and antibodies. Cochleas from 21 guinea pigs were used in this study and were prepared either by an immunofluorescence technique for confocal imaging of GLAST $(n=12)$, GluR4 $(n=6)$, and VGLUT1 $(n=$ 4) labeling or embedded for postembedding immunogold labeling for transmission electron microscopy (TEM) $(n=5)$. We also attempted to label for VGLUT2 $(n=2)$, another vesicular glutamate transporter, which differs from VGLUT1 in distribution in the brain (Bai et al., 2001). Animals were maintained and used in accordance with the Principles of Laboratory Animal Care (NIH publication 85-23, revised 1983) and the UK Animals (Scientific Procedures) Act, 1986.

A polyclonal antibody to GLAST raised against a synthetic peptide based on C-terminal residues 522-541 (sequence PYQLIAAQDNEPEKPVADSET) from rat (Storck et al., 1992) was kindly provided by N. C. Danbolt and K. P. Lehre (Preclinical Institute, University of Oslo, Oslo, Norway) (Lehre et al., 1995). VGLUT1 and VGLUT2 antibodies were purchased from Synaptic Systems (Göttingen, Germany). The VGLUT1 polyclonal antibody was raised against recombinant glutathione $S$-transferase (GST)-fusion protein containing amino acid residues $456-$ 560 of rat VGLUT1. The VGLUT2 polyclonal antibody was raised against a recombinant GST-fusion protein containing amino acid residues $510-$ 582 of rat VGLUT2. A GluR4 polyclonal antibody was obtained from Sigma (Poole, Dorset, UK) and raised against a synthetic peptide (sequence SEVDKMVVTLTEL) from the C-terminal region of rat GluR4. Fluorochrome-conjugated secondary antibodies were obtained from Sigma, and gold-conjugated secondary antibodies were obtained from British Biocell International (Cardiff, UK).

Fixation of the cochlea and cerebellum. Guinea pigs were anesthetized with a $1 \mathrm{ml} / \mathrm{kg}$ intraperitoneal injection of sodium pentobarbitone (Pentaject; VetDrug) and decapitated once the pedal withdrawal reflex was lost. To expose the cochlea, the lower jaw was removed and the bullas were excised and opened. For immunofluorescence, fixative consisting of freshly dissolved paraformaldehyde, $4 \% \mathrm{w} / \mathrm{v}$ in $0.1 \mathrm{~m}$ sodium phosphate buffer (NaPi), pH 7.4, was perfused through holes made in the apex, and the round window of the cochleas and the bullas were then immersed in this fixative for $2 \mathrm{hr}$ on a rotator. To act as a control tissue for fluorescence labeling, portions of cerebellum, where GLAST is known to be highly expressed (Lehre et al., 1995; Lehre and Danbolt, 1998), were also excised from five guinea pigs once the bulla had been removed, and sliced thinly using a razor blade; the slices were then immersed in the same fixative.

For TEM, glutaraldehyde was added to the formaldehyde fixative described above to make $0.1 \% \mathrm{v} / \mathrm{v}$ that was perfused through the cochlea as before.

Immunofluorescent labeling and confocal microscopy. For immunofluorescent labeling of the cochlea, the shell was removed, the modiolus broken across at its base, and the entire spiral lifted out. This procedure resulted in preservation of the top three to four of the 4.5 turns of the cochlear spiral, but loss of the organ of Corti in the hook and to a variable extent, the lower basal turn.

The tissue was first washed in $\mathrm{NaPi}$ and then incubated in $0.1-0.5 \%$ $\mathrm{v} / \mathrm{v}$ Triton X-100 in PBS for $30 \mathrm{~min}$, washed in PBS, blocked in 10\% v/v goat serum (GS) in PBS for $30 \mathrm{~min}$, incubated for $2 \mathrm{hr}$ at room temperature or overnight at $4^{\circ} \mathrm{C}$ in $5 \mu \mathrm{g} / \mathrm{ml}$ anti-GLAST, 1:50-1:100 antiGluR4, 1:100 anti-VGLUT1, or 1:100 anti-VGLUT2 in PBS containing $1-2 \% \mathrm{v} / \mathrm{v}$ GS (GS-PBS), and then washed in PBS. Cochleas were then incubated in FITC- or tetramethylrhodamine isothiocyanate (TRITC)conjugated goat anti-rabbit secondary antibody in 1-2\% GS-PBS for $1 \mathrm{hr}$ and finally washed before dissection. The spiral was dissected into segments that were mounted in an antifade solution consisting of $0.01 \% \mathrm{w} / \mathrm{v}$ p-phenylene diamine in a 30\% v/v glycerol/30\% v/v polyvinyl alcohol solution.

Additionally, in those animals in which cerebellum was also fixed, $100-\mu \mathrm{m}$-thick sections of the cerebellar portions were cut on a Leica VT1000s Vibratome and labeled simultaneously with the cochlear spiral in the same wells for GLAST using the above protocol. The cerebellar slices were mounted on the same slides as the cochlear segments from the same animals so that they could be imaged under identical conditions.

In some cochleas in which the secondary antibody used was TRITCgoat anti-rabbit, an FITC-conjugated anti-tubulin antibody that recognizes the $\mathrm{C}$ terminal (Sigma) was included with the secondary antibody (dilution 1:100). The tubulin labeling acted as an indicator of hair-cell shape and a check for possible artifactual variations in fluorescence.

Cochlear segments and cerebellar slices were examined in a Bio-Rad MRC1024 multiphoton microscope in confocal mode using a kryptonargon laser with excitation wavelengths of $488 \mathrm{~nm}$ (for FITC) and $567 \mathrm{~nm}$ (for TRITC). To detect gradients in the levels of protein expression along the cochlea, sequential images of all segments were taken in bright-field mode using a $10 \times$ objective so that the location of the areas acquired for analysis could be determined with respect to the apex. During acquisition of images for measurement, attenuation of the laser and gain of the photomultiplier tubes were kept constant for each cochlea. A set of 20-30 fluorescent image planes with z steps of $1 \mu \mathrm{m}$ was then acquired from each of $8-12$ regions spaced approximately equally along the cochlea using a $60 \times$ objective. Apart from distance from the apex, choice of these locations was determined mostly by the extent to which a clear view of a substantial portion of the IHC row could be observed in bright-field microscopy.

Measurements of the intensity of fluorescent labeling for GLAST, VGLUT1, and, where appropriate, tubulin were made by adding each set of images vertically in the Bio-Rad software and drawing a line through the row of IHCs, at the level of their nuclei, from which the mean pixel intensity of the labeling was derived. The length of this line was similar, although not identical, in all cases and determined by the extent of the IHC row within the image and whether there were areas of disturbance to avoid. Repeated measurements along lines of varying length within a single image indicated that these minor variations did not affect the mean value of pixel intensity. Comparative measurements of cerebellar and cochlear GLAST labeling were made along lines drawn across single images of the molecular layer of the cerebellum and from IHC regions of the cochlea imaged under identical conditions after simultaneous labeling. Single images were used because the labeling was confined to a narrow layer near the surface of the cerebellar slices.

We also performed controls to determine the extent to which differences in fluorescence intensity accurately reflect relative differences in GLAST concentration. For this purpose we excised portions of cerebellum from four guinea pigs, homogenized them in sample buffer composed of $62.5 \mathrm{~mm}$ Tris- $\mathrm{HCl}$, pH 6.75, containing 2\% SDS, 5\% mercaptoethanol, $10 \%$ glycerol, and $0.001 \%$ bromophenol blue $(\sim 0.18 \mathrm{mg}$ cerebellum tissue per milliliter), and then boiled them for $3 \mathrm{~min}$. An equal volume of PBS was added to produce stock solutions that were diluted from 100 to $10 \%$ in steps of $10 \% ; 0.2 \mu \mathrm{l}$ volumes of each sample in the series were then dot-blotted in sequence onto strips of nitrocellulose that were labeled for GLAST. They were washed in PBS, incubated in $10 \% \mathrm{v} / \mathrm{v}$ GS in PBS (10 min) and then in anti-GLAST antibody $(5 \mu \mathrm{g} / \mathrm{ml}$ in $1 \% \mathrm{v} / \mathrm{v}$ GS-PBS) overnight, washed in PBS, and incubated in FITCconjugated goat anti-rabbit secondary antibody in 1-2\% GS-PBS for 2 hr. The strips were mounted on slides in PBS and examined in the BioRad MRC 1024 microscope. The dots were viewed using a 10× objective, and the attenuation of the laser and gain of the photomultiplier were adjusted to produce a mean pixel intensity of $\sim 70$ (measured along a randomly chosen radius through the dot) on the $100 \%$ dot. In using these conditions, each dot was then examined, and either a stack of images or the single brightest image plane was acquired. A mean intensity was then determined for each along a randomly selected radius.

To investigate the distribution of GluR4 labeling, which took the form of puncta localized mainly to the IHC base, we counted the number of labeled sites. Three consecutive images corresponding to this region were selected at low magnification to reduce any selection bias, the middle image was omitted to avoid double counting the same puncta, and a fixed 
area was drawn on the remaining panes containing five IHCs. The number of labeled puncta was then counted at high magnification. This method meant that a small number of outlying puncta in unselected planes were not included in these counts. In addition, variation in labeling intensity in different cochleas also may have affected the estimate by reducing the number of visible puncta.

Preparation for postembedding immunogold labeling for electron microscopy. Five cochleas were fixed for TEM and dissected to remove a small amount of the shell. They were then dehydrated in an ethanol series and infiltrated with LR White hard grade resin (Agar Scientific, Stansted, UK) via four changes (durations: $1 \mathrm{hr}$, $1 \mathrm{hr}$, overnight, $1 \mathrm{hr}$, respectively) under vacuum. The cochleas were then placed into gelatin capsules and immersed in fresh resin that was polymerized at $50^{\circ} \mathrm{C}$ for $24 \mathrm{hr}$.

Embedded cochleas were bisected to produce hemicoils using a Microslice 2 rotary cutter (Malvern Instruments, Malvern, UK) with an annular diamond blade (Jiang et al., 1993). U1trathin $(\sim 120 \mathrm{~nm})$ sections of the organ of Corti were cut in a radial plane from regions along the spiral located 1 and $10 \mathrm{~mm}$ from the apex. The sections were collected onto nickel grids coated with quick-drying adhesive (Coatquick "G"; Agar, Stansted, UK).

For immunolabeling, grids were incubated sequentially in drops of the following solutions (in a moist chamber and, unless stated otherwise, at room temperature): $0.05 \mathrm{M}$ Trisbuffered saline (TBS), pH 7.4 (5 $\mathrm{min}$, three times); $50 \mathrm{~mm}$ glycine in TBS (10 min); 20\% v/v GS-TBS to block nonspecific binding (30 min); primary antibody (either anti-GLAST diluted to $1-5 \mu \mathrm{g} / \mathrm{ml}$ or anti-GluR4 diluted $1: 100)$ in
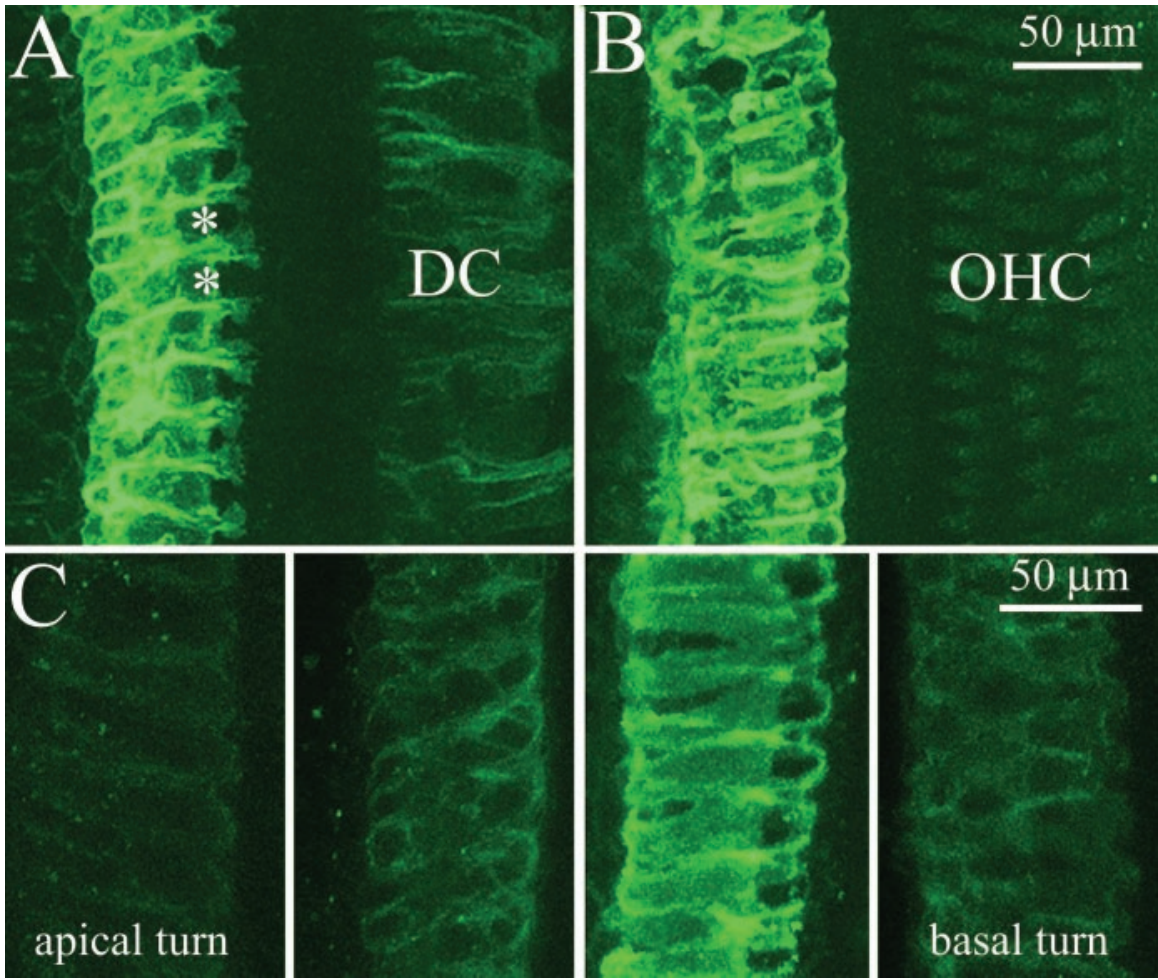

Figure 1. Confocal image stacks of the guinea pig organ of Corti after immunofluorescent labeling for GLAST.A, Apical turn. The fluorescent labeling can be seen in supporting cells surrounding the unlabeled flask-shaped IHCs (*) and more weakly in the membranes of the Deiters' cells (DC). B , Basal turn. The distribution of labeling in the IHC supporting cells is very similar to that seen in the apical region, but labeling in the Deiters' cell membranes is absent. Background fluorescence can be seen in the OHCs. Note that the fluorescence from supporting cells around the IHCs is saturated in intensity because the power of the laser and gain of the photomultiplier were set high to detect Deiters' cell labeling. C, Sequence of images of the IHC region from each turn of the cochlear spiral progressing from the apex to the base. Here the power and gain were kept low to allow variations in the intensity in the IHC supporting cells to be detected. Note that qualitatively, there appears to be an increase in fluorescence intensity toward the base, but this decreases again in the most basal image. $1 \% \mathrm{v} / \mathrm{v}$ bovine serum albumin in TBS (BSA-

TBS) at $37^{\circ} \mathrm{C}$ for $2 \mathrm{hr}$; BSA-TBS ( $5 \mathrm{~min}$, three times); GS-TBS (10 min); 10- to 30-nm-diameter gold particles conjugated to goat anti-rabbit IgG diluted 1:20 in BSA-TBS (1 hr); TBS (5 min, three times); and distilled water ( $5 \mathrm{~min}$, two times). Finally, sections were stained in $2 \%$ ethanolic uranyl acetate, washed in distilled water, and observed using a JEOL 100CX transmission electron microscope operated at $100 \mathrm{kV}$.

Controls were performed either by omitting the antibody or by replacing it with rabbit IgG at the same concentration. No specific labeling was found in these controls.

Semiquantitative analysis of immunogold labeling. Levels of GLAST labeling density were compared between the 1 and $10 \mathrm{~mm}$ distances from the apex and between the pillar side and the modiolar side of the IHC. A minimum of three separate sections of the base of IHCs were analyzed from each location in each animal. Micrographs of the base were taken, and the cell was divided longitudinally by a line through its center to separate the pillar-cell and modiolar sides. The lengths of cell membranes of the supporting cells on each side were measured, and the number of particles along them was then counted and the labeling density calculated.

\section{Results}

Distribution of GLAST along the length of the guinea

pig cochlea

In whole mounts of guinea pig cochlear segments, immunofluorescent labeling for GLAST could be clearly observed by confocal microscopy. Labeling was strongest in the IHC region where it outlined the shape of the IHCs, consistent with the presence of GLAST in the surrounding inner phalangeal and border cells. In apical regions of the guinea pig cochlea, much weaker labeling of
Deiters' cell membranes could also be detected if the confocal laser power and photomultiplier gain were high, but this was absent in basal regions (Fig. 1A,B). When imaged under conditions appropriate to view Deiters' cell labeling, the fluorescent intensity in supporting cells around the IHCs was saturated so that no differences with location were visible.

By increasing laser attenuation and reducing photomultiplier gain, it was possible to detect differences in GLAST labeling intensity in supporting cells associated with IHCs in different locations. Imaging of the different turns of the guinea pig cochlea under constant conditions revealed consistent qualitative differences in labeling intensity of the inner phalangeal and border cells, with labeling intensity being higher nearer the mid-regions of the cochlea than at either end (Fig. 1C). We thus explored the possibility that fluorescent intensity could be used to estimate the relative changes in the concentration of GLAST.

To do this we produced dot blots on nitrocellulose of extracts of cerebellum diluted in steps of $10 \%$ and labeled them for GLAST. From the weight of the cerebellum samples that were collected and the fixed ratio of sample buffer volumes that was used, the stock solution obtained from each animal contained $\sim 0.18 \mathrm{mg} / \mathrm{ml}$ tissue. Lehre and Danbolt (1998) have determined that the amount of GLAST in whole cerebellum preparations is $0.74 \mathrm{mg} / \mathrm{g}$. From this, assuming complete solubilization, we estimate that the concentration of GLAST in the extracts used for dot blotting ranged between $\sim 130 \mathrm{ng} / \mathrm{ml}$ in the $100 \%$ stock solution and $\sim 13 \mathrm{ng} / \mathrm{ml}$ in the $10 \%$ solution. 


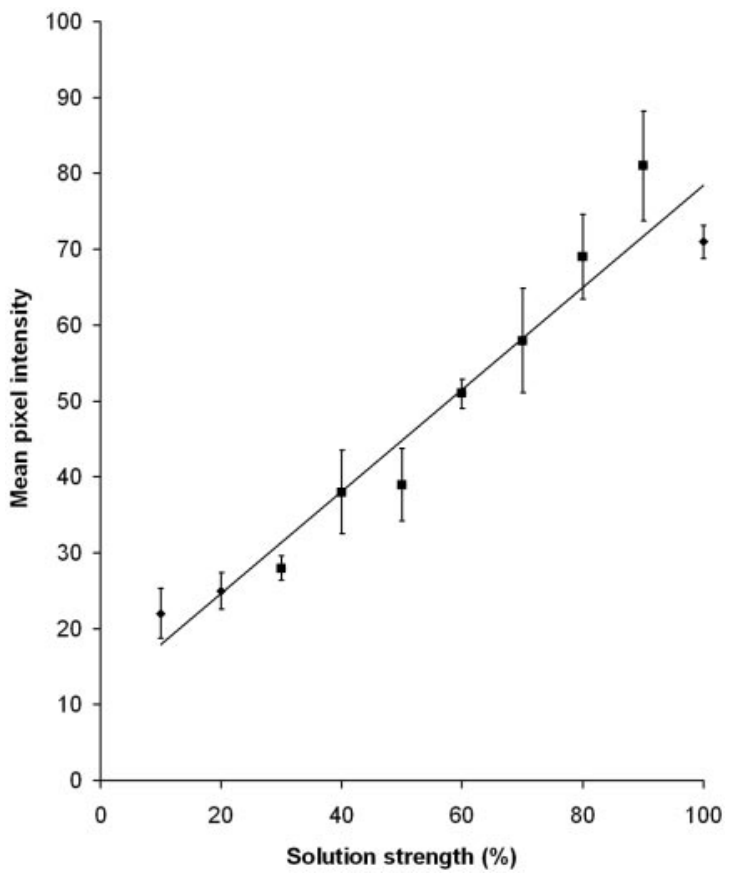

Figure 2. Graph showing how fluorescent intensity (measured as mean pixel intensity along a line through the image) of dot blots of extracts from cerebellar tissue labeled for GLAST varies with relative GLAST concentration, expressed as a percentage of the stock solution. Mean values were obtained by combining data from extracts from four animals. For each of the four sample series, the confocal settings were adjusted to give a mean pixel intensity of $\sim 70$ for the $100 \%$ dot, and then all subsequent dots of the series were imaged under the same conditions. With the exception of the 10,20 , and $100 \%$ dots, the values vary linearly with relative solution strength. (Filled squares represent those values that lie in the linear range; filled diamonds represent those that lie outside it.)

We imaged the dots and determined mean fluorescent intensities from four preparations under comparable conditions (Fig. 2 ). This showed that the fluorescent intensity changes in proportion to the relative change in concentration of GLAST over the majority of the dilution series. Under these conditions, the least concentrated dots $(10,20$, and $30 \%)$ showed relatively small increments and were close to background. The highest concentration $(100 \%)$ was weaker in intensity than the $90 \%$ dot, suggesting inhibition of the labeling in high protein concentrations; however, in the 30-90\% range the relationship was linear $\left(R^{2}=0.98\right)$ and the gradient close to unity (0.9). Thus, provided the signals are not saturated or very weak, fluorescent intensity does seem to reflect relative changes in GLAST levels. In our dot blots the linear range represents a change in GLAST concentrations of $\sim 120$ to $\sim 40 \mathrm{ng} / \mathrm{ml}$, or a threefold change. A similar graph was obtained if either the single brightest image plane or stacks of images were used.

When the laser power and photomultiplier gain were increased to enhance the fainter dots, a greater difference between the 10 and 20\% dots became apparent (data not shown). Under these circumstances, however, the dots representing more concentrated solution became overwhelmingly intense so that the majority of the signal was saturated and differences between them could no longer be detected. We concluded that, provided the regions of maximum and minimum intensities of immunofluorescence labeling were both imaged and the settings adjusted to establish the optimal signal (i.e., with no saturation of pixel intensity at the higher end and with clear signal above background at the lower end), relative changes of concentration of up to threefold would be well represented by intensity measurements.
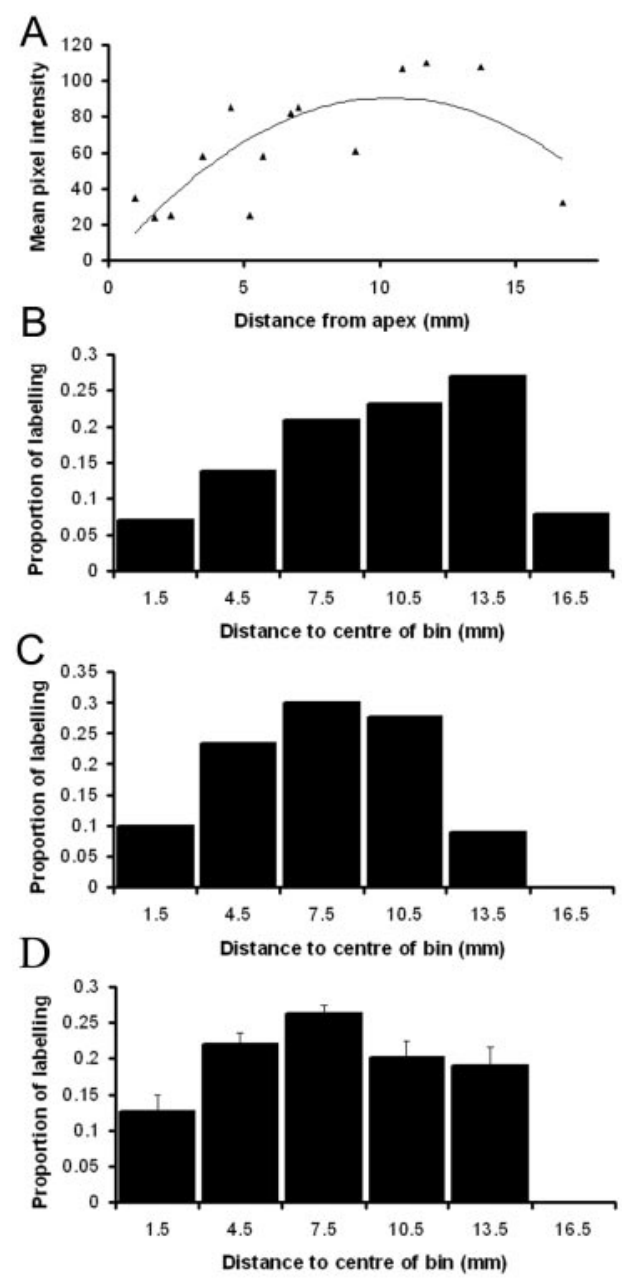

Figure 3. Graphs and histograms showing how the mean fluorescent intensity for GLAST labeling varies with position along the cochlea. $A$, Raw data from one cochlea in which the mean pixel intensity in each set of images was measured for a line drawn along the IHC row. Note that although there is variation in fluorescence intensity, the overall trend shows a progressive increase toward the mid-basal region of the cochlea. A second-order polynomial trend line has been added to show the overall pattern of the data points. $B$, Histogram of mean fluorescence intensity in $3 \mathrm{~mm}$ bins calculated as a proportion of the total fluorescence intensity, from the data shown in $A$. This confirms that the labeling gradually increases toward the middle region but then decreases again toward the base. $C$, A second example of a single cochlea showing a pattern of labeling similar to that observed in $B$, although peaking more apically. D, Data plotted as in $B$ but representing mean values from the cochleas of seven animals. Note that the peak of fluorescence intensity occurs in the $7.5 \mathrm{~mm}$ bin, after which it tends to decline. Bins after $15 \mathrm{~mm}$ have been omitted because this region was rarely preserved. Error bars show the SE of the means.

We also compared fluorescent labeling intensity in cerebellar slices with the labeling intensity in the cochlea. Stronger fluorescence was noted in the cerebellar molecular layer than in any region of the cochlea. We therefore compared the most heavily labeled region of the latter to avoid the problems of nonlinearity encountered in the extreme range of concentrations in the dot blots. Mean labeling intensity associated with the IHCs of the mid-region cochlea was $60 \pm 20 \%( \pm$ SD; $n=5)$ of the labeling intensity in cerebellum.

It is important, however, to be cautious about these relative measures in different tissues because they will reflect not only concentrations of GLAST but also differences in quantity and distribution of membranes containing GLAST. Thus, although 
we have estimated protein concentrations in the dot solutions, in the present work we have not attempted to use these values to estimate or directly compare the concentration of GLAST protein in tissues. This is because in the tissue the GLAST is mostly confined to membranes (see below), whereas in the dot blots it is distributed homogeneously.

Nevertheless, our data suggested that we could quantify relative changes in GLAST labeling along each cochlea using fluorescence measurements. We therefore determined the mean pixel intensity of the labeling along a line through the IHC row from each location. Although the raw data showed some variability, a clear gradient in fluorescent intensity was noted rising toward the mid-basal region of the cochlea (Fig. 3A). Because of the variability in precise location of measurements (see Materials and Methods), we could not combine fluorescence measurements from the different samples directly as was done for the dot blots. Instead, fluorescent intensity measurements were standardized by collecting values in bins of $3 \mathrm{~mm}$ cochlear length, finding the mean fluorescent intensity per bin, and then dividing each of these values by the total of the mean intensities within each animal to obtain a proportion of labeling per bin (Fig. $3 B, C$ ). These proportional data were combined from several animals $(n=7)$ to look for overall trends. This confirmed the gradient in fluorescent intensity and showed that it rises to a peak in the $7.5 \mathrm{~mm}$ bin (Fig. 3D). The mean proportional fluorescence intensity in the $7.5 \mathrm{~mm}$ bin was significantly different from the intensity in either apical or basal bins (Kruskal-Wallis test, followed by Man$n-$ Whitney $U$ test on individual pairs; $p>0.05$ ).

To provide further confirmation that there is a change in the density of GLAST in the membrane, immunogold labeling was performed on radial sections cut from $\sim 1$ and $10 \mathrm{~mm}$ regions (measured from the apex). These regions were selected for comparison because the preliminary fluorescence measurements suggested that there was a substantial difference between them, and they could be readily separated for TEM using the microslicing technique that we have used. The immunogold labeling was present mainly on the inner phalangeal-cell and border-cell membranes (Fig. 4).

Analysis of gold particle density on the supporting-cell membranes in radial sections from each location in five animals revealed systematic differences with position. The ratio of labeling between the 10 and $1 \mathrm{~mm}$ points was $>1$ for each animal because labeling density was always greater in the sections from the 10 $\mathrm{mm}$ point (Fig. 5A). These differences were significant (Wilcoxon signed rank test; $p<0.05$ ). The mean ratio between gold labeling density in the 1 and $10 \mathrm{~mm}$ regions was 1.4 , which is comparable with the equivalent ratio in the fluorescence intensity between these two points, which was 1.5 (Table 1).

\section{Distribution of GLAST around the IHC base}

Because it has been reported that higher SR fibers synapse on the pillar-cell side of IHCs, we compared the amount of gold labeling on either side of the hair-cell base. Gold labeling appeared qualitatively stronger on the pillar-cell side (Fig. 4, compare $B, C$ ). To determine whether this difference was systematic, profiles of supporting cells were classified as either pillar side or modiolar side, and the gold particle density in their membranes was measured in the same sections from the five animals described above. The ratio of pillar to modiolar side labeling density was then calculated (Fig. 5B). The mean pillar/modiolar ratio in the five animals was always $>1$ in both the 1 and $10 \mathrm{~mm}$ regions, and the overall mean ratio was 2.55 . The difference in density between the pillar and modiolar side was highly significant (Wilcoxon signed rank test; $p<0.001$ ). There was, however, no significant difference between the ratios obtained for the 10 and $1 \mathrm{~mm}$ regions when these were calculated separately.

\section{Distribution of GluR4 along the length of the guinea pig cochlea}

In immunofluorescence, GluR4 labeling was present as small, well defined puncta in the IHC region. Double labeling for tubulin and GluR4 showed that the latter tended to occur near the 

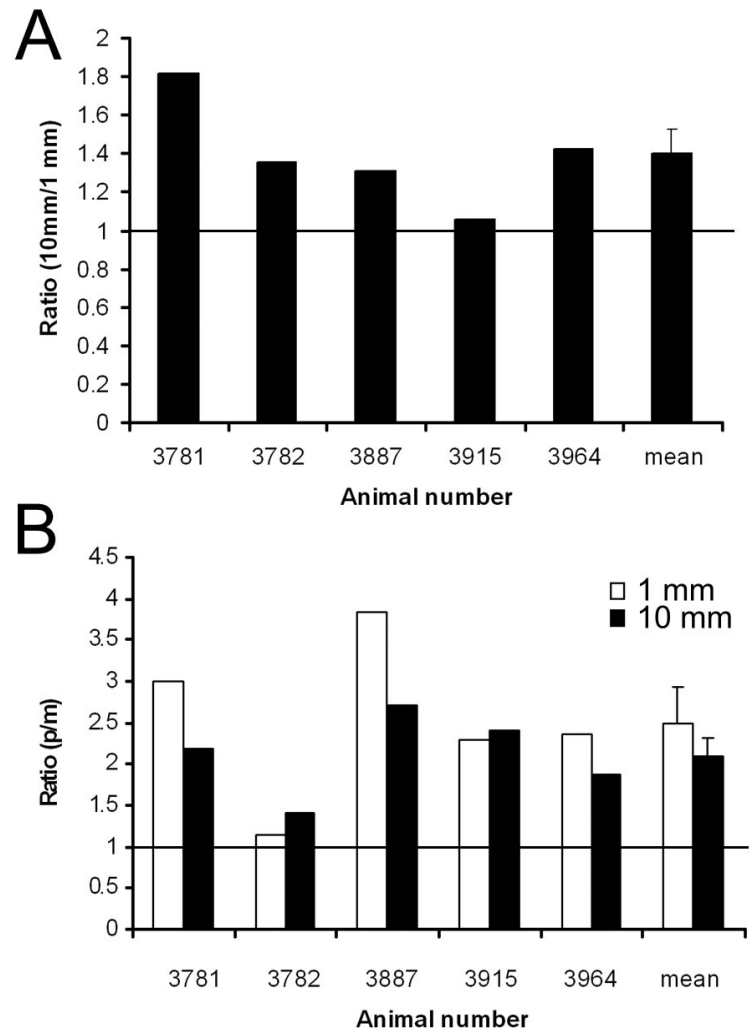

Figure 5. A, Histogram showing the ratio of gold labeling density in supporting cells of the $I H C$ region between the 10 and $1 \mathrm{~mm}$ points for five animals, and the mean ratio and SE of the five. Note that the ratio is always higher than 1 (as indicated by the horizontal line). $B$, Histogram showing the ratios of labeling between the pillar cell and the modiolar side in the $1 \mathrm{~mm}$ (white bars) and the $10 \mathrm{~mm}$ (black bars) region for five animals, and the mean ratio and SE of the five. The ratio is always $>1$ (shown by the horizontal line), indicating that the labeling density is higher on the pillar-cell side than the modiolar side in both locations.

Table 1. Ratios comparing the 1 and $10 \mathrm{~mm}$ regions for immunofluorescent intensity and gold labeling density for GLAST, and numbers of GluR4 puncta

\begin{tabular}{ll}
\hline Comparison & $10 \mathrm{~mm} / 1 \mathrm{~mm}$ ratio \\
\hline Fluorescence intensity & 1.5 \\
Immunogold labeling density & 1.4 \\
GluR4 puncta & 1.3 \\
\hline
\end{tabular}

Note the similarity of the three ratios.

hair-cell base (Fig. 6A-C), although weak fluorescence could be detected around the apical cuticular plate. It was apparent that the overall number of puncta was greater in the mid-basal cochlear region than in either apical or most basal regions (Fig. 6D).

Examination of sections by TEM after postembedding labeling with the GluR4 antibody revealed that gold particles were confined primarily to the region of synaptic density and were most often closest to the presynaptic membrane, adjacent to the dense bars where the afferent terminal contacted the base of IHCs (Fig. 7), rather than to the postsynaptic membrane. Thus the puncta in the immunofluorescent samples probably represent single synaptic densities. In one animal, 74 puncta were counted in the $1 \mathrm{~mm}$ region and 114 in the $10 \mathrm{~mm}$ region, in each case in an area representing five IHCs. This indicates a difference in number from 15 per IHC in the $1 \mathrm{~mm}$ region to 23 per IHC in the $10 \mathrm{~mm}$ region. Because the numbers of puncta were lower than this in the other animals, however, it is possible that either not all sites are labeled or our counting method underestimates the total number of synaptic sites in some cases.
Despite the inter-animal variability, changes in the number of puncta along a single cochlea are likely to reflect relative changes in number of synaptic sites. Thus, to determine whether there was any gradient in distributions of the AMPA receptor sites, the number of puncta per fixed area was determined in images taken at different points along the length of the cochlea (Fig. $8 \mathrm{~A}$ ). This showed a peak in number of puncta in the mid-region of the cochlea in six animals. To standardize the labeling, we divided the cochlea into bins as before and determined the proportional distribution by dividing the individual values per bin by the total number of puncta (Fig. $8 \mathrm{~B}$ ). When data from all six animals were combined, this revealed a gradient that was very similar to that observed for GLAST (Fig. 8C). The proportion of labeled puncta was significantly higher in the $7.5 \mathrm{~mm}$ bin than in the $1 \mathrm{~mm}$ bin (Mann-Whitney $U$ test; $p<0.05 \%$ ). For comparison with the immunogold labeling density (1.4) and fluorescence intensity (1.5) ratios between the 1 and $10 \mathrm{~mm}$ regions, the mean ratio of numbers of puncta between the same regions is 1.3 (Table 1).

Distribution of VGLUT1 and VGLUT2 along the length of the guinea pig cochlea

Immunofluorescent labeling for VGLUT1 was detected by confocal imaging and was confined to the IHC region. It consisted of small, somewhat poorly defined patches of fluorescence within the IHC cytoplasm, particularly toward the base of the hair cell and alongside the membrane (Fig. 9A), and in the region of the inner spiral bundle (Fig. 9B). Although no gradients in fluorescent intensity were observed qualitatively (Fig. 9B), we measured fluorescent intensity at different cochlear locations as described above for the GLAST labeling. This did not show a peak in labeling intensity (Fig. 10A). Postembedding labeling with this antibody was not successful. No labeling was observed with the antibody to VGLUT2.

\section{Distribution of tubulin}

As expected, the FITC-anti-tubulin labeled supporting cells and hair cells and acted as a marker for IHC bodies for GluR4 labeling (Fig. $6 A, C$ ). It also provided a check that the gradients in GLAST were not artifacts of preparation that were caused, for example, by systematic variations in antibody penetration. Measurement of fluorescent intensity along a line drawn through the IHCs at different points along the cochlea showed a very different pattern from the GLAST labeling. The data tended to be quite variable, and there was no clear peak in the mid-region (Fig. 10B).

\section{Discussion}

We have described corresponding gradients in expression of the glutamate transporter GLAST and number of GluR4-labeled puncta in the cochlea and an association between local GLAST distribution and synaptic activity. We have also detected VGLUT1 in IHCs and possibly the inner spiral bundle, but no gradient in expression along the cochlea.

In the CNS, GLAST expressed in glial cells contributes significantly to glutamate uptake, which may help to terminate synaptic action and protect neurons from the excitotoxic effects of elevated extracellular glutamate (for review, see Danbolt, 2001). In the cochlea, mice lacking GLAST contain higher baseline levels of glutamate than normal mice, supporting a role in glutamate uptake. Moreover, after acoustic overstimulation, there is greater evidence of excitotoxicity in the afferent terminals than in normal mice, suggesting that GLAST protects them from glutamate overrelease (Hakuba et al., 2000).

Given that GLAST is probably involved in glutamate handling 
in the cochlea, we have made use of systematic variations in innervation along the cochlear spiral to explore factors involved in its regional expression. We have identified two aspects of the innervation that appear to be related to GLAST expression: (1) the number of synapses and (2) the activity level of synapses.

With respect to the number of synapses, the gradient in immunofluorescent GLAST labeling in IHC supporting cells coincides with a gradient in the number of GluR4-labeled puncta, which immunogold labeling suggests represents synaptic sites. Both reach a peak $\sim 6-9 \mathrm{~mm}$ along the guinea pig cochlea. In vivo, the peak may be more basal than this, because tissue shrinkage during fixation and losses during dissection could lead us to underestimate the length (Jiang et al., 1993). Differences in GLAST expression have also been detected using the immunogold technique, and there are similar proportional increases in the immunofluorescent intensity measurements and the density of gold labeling between the 1 and the $10 \mathrm{~mm}$ regions.

It is important to address the extent to which the fluorescent gradients that were detected correspond to real differences in protein expression. Our control series of
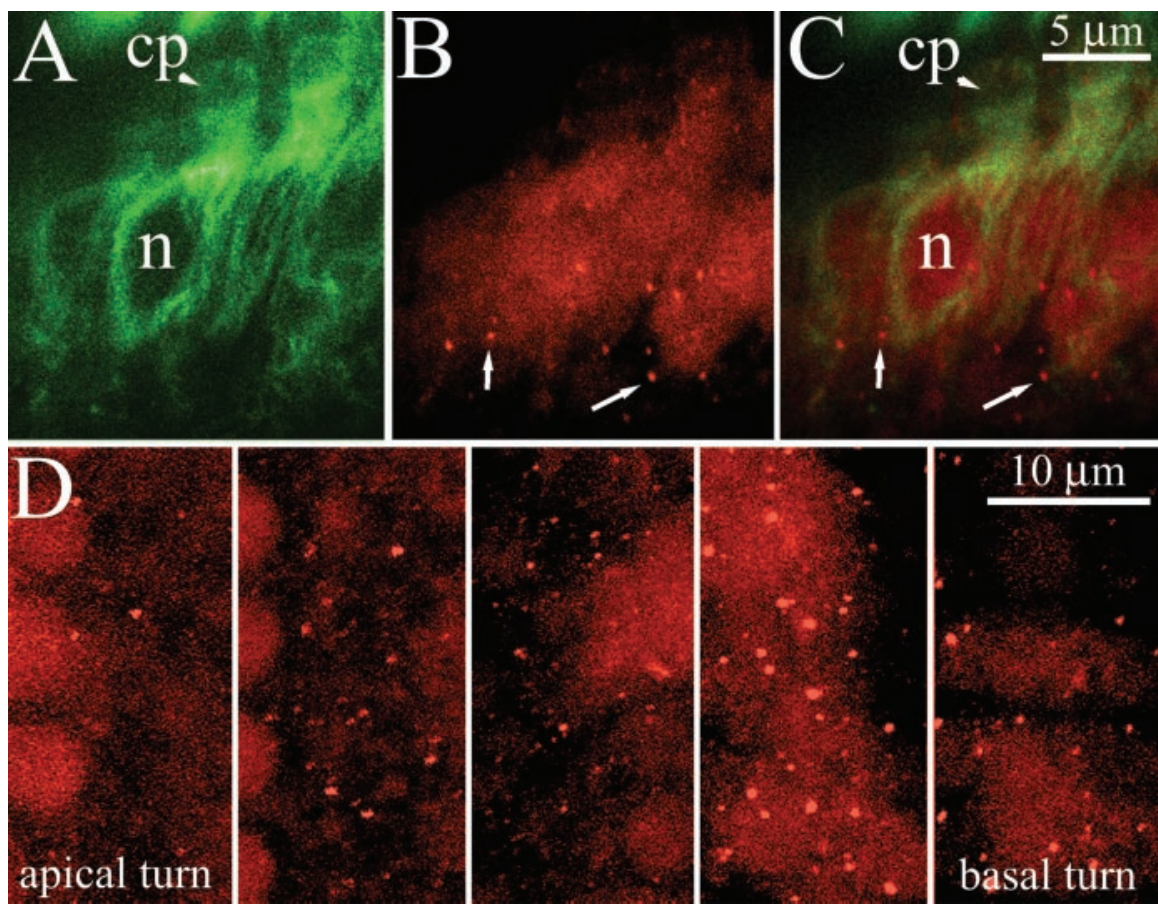

Figure 6. Immunofluorescent labeling of GluR4 receptor subunits and tubulin in the IHC region. $A-C$, Three images of the same IHCs double labeled for tubulin (green) and GluR4 (red). $A$, Tubulin labeling showing the flask-shaped IHC bodies, with microtubules outlining the region of the nucleus ( $\mathrm{n}$ ) and apical cuticular plate (cp). $B$, GluR4 labeling showing that puncta (arrows) are confined primarily to the basal end of the hair cell (as defined by the tubulin labeling shown in $A$ ). $C, A$ and $B$ combined. $D$, Sequential images of the IHC region along the cochlear spiral showing an increase in number of GluR4 puncta to a peak in the mid-basal cochlear region. dot blots showed that, provided appro-

priate settings are adopted for confocalimaging, a good correspondence can be obtained between relative GLAST concentrations and fluorescent intensity. Deviations from linearity occurred only at the lowest and highest concentrations. This may be influenced not only by the laser settings but also by limitations in the dot-blotting experiment in which a wider range of GLAST concentrations were used than is likely to occur in the cochlea; for example, steric hindrance at high protein concentrations may inhibit antibody access to epitopic sites.

It is possible that the immunofluorescent intensity gradients observed could result from the systematic changes in tissue thickness that occur along the cochlear length (Jiang et al., 1993), affecting the degree of antibody penetration. This seems unlikely, however, because there was no equivalent gradient in either VGLUT1 or tubulin labeling, whereas an opposite gradient was noted in GLAST labeling of Deiters' cells associated with OHCs (see below).

Similarly, it is necessary to assess the extent to which the number of GluR4 puncta reflects innervation density. Although nerve fiber counts by light microscopy showed little evidence of gradients in IHC afferent innervation density in guinea pig (Morrison et al., 1975), Liberman et al. (1990) found in cat using TEM that numbers of synapses increase from the apex to a peak and then decline basally, similar to changes in the number of GluR4 puncta found here in guinea pig. Previous TEM studies in guinea pig are less complete, but synaptic counts from apical and mid-basal locations (Hashimoto et al., 1990) compare well with the relative numbers of GluR4 puncta overall and with absolute numbers in one animal. In the latter, in the apical region there were 15 puncta per IHC compared with 17 synapses counted by TEM, and in the mid-basal region there were 23 puncta compared with 26 syn-

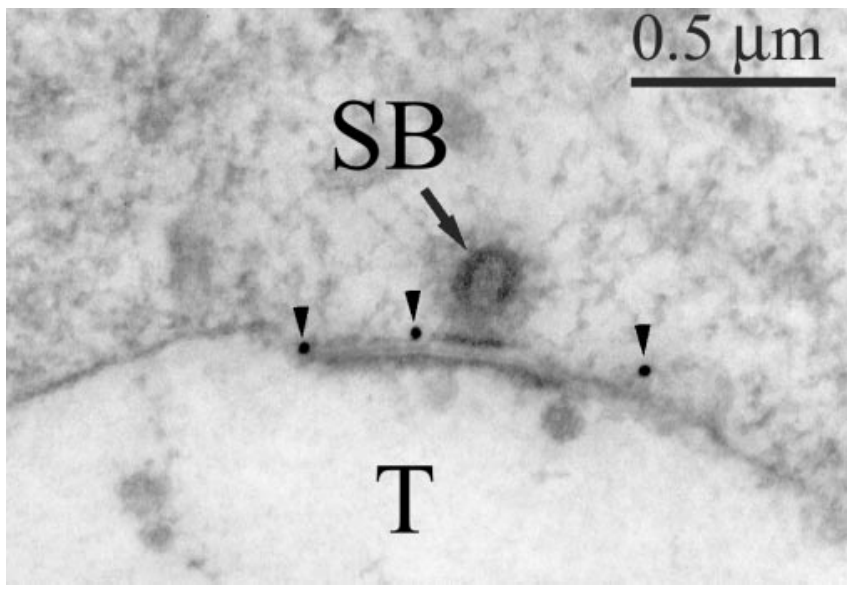

Figure 7. Immunogold labeling with GluR4 antibody. Note that gold particles (arrowheads) occur mostly near the presynaptic membrane adjacent to a synaptic bar (SB) in the IHC and postsynaptic densities on the afferent nerve terminal $(\mathrm{T})$ membrane.

apses. In the other animals, the fewer puncta found may reflect weaker levels of labeling.

It may be possible to confirm these patterns by detecting other proteins in IHC synapses; however, GluR4 seems the most suitable candidate because it appears to be restricted to these synapses, whereas other proteins occur elsewhere. For example, in an extensive study of postsynaptic density (PSD) proteins, Davies et al. (2001) found that one antibody to PSD-95 not only labeled puncta around IHCs but also nerve fibers, whereas other PSD-95 antibodies produced no labeling; PSD-93 antibodies labeled more extensively around the IHC base. Synaptophysin antibodies label only the efferent system (Counter et al., 1997). GluR2/3 


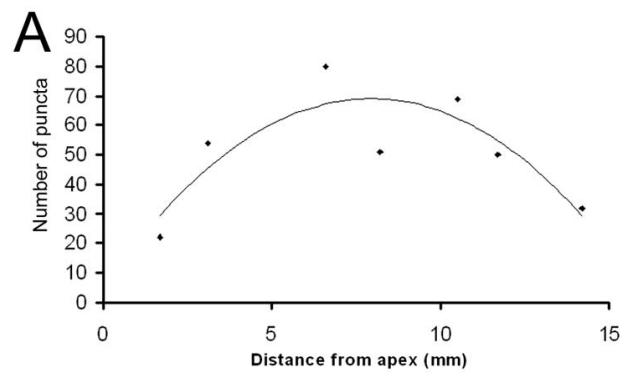

B
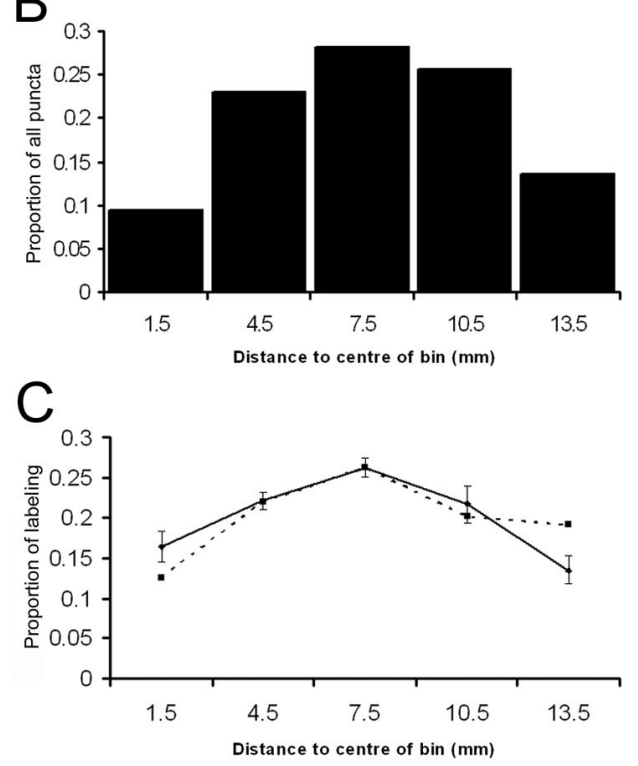

Figure 8. Graphs and histograms showing how the relative number of fluorescent puncta for GluR4 labeling associated with IHCs varies with position along the cochlea. A, Raw data from one cochlea in which the number of puncta was determined from two images of the synaptic region of five IHCs. Note that there appears to be a progressive increase in number toward the mid-basal region of the cochlea. A second order polynomial trend line has been added to show the overall pattern of data points. $B$, Proportions of puncta expressed in $3 \mathrm{~mm}$ bins from the data shown in A. C, Data plotted as in $B$ but obtained from the cochleas of six animals (solid line). Note that the peak occurs in the $7.5 \mathrm{~mm}$ bin after which the number of puncta tends to decline. Error bars show the SE of the means. The GLAST data from Figure 2 are superimposed (dashed line) to show the correspondence between the two. Apart from the most basal point, the data show good agreement.

receptor subunits are localized to the same synapses as GluR4 (Ottersen et al., 1998); hence labeling for them is likely to be similar.

The comparability of our findings from confocal microscopy and TEM, and the lack of similar patterns in VGLUT1 or tubulin, suggest that the gradients are real, not artifactual, and represent corresponding changes in supporting-cell expression of GLAST and the number of GluR4 puncta at the IHC base. An equivalent correspondence has been found between GLAST in Deiters' cells and the afferent innervation to the OHCs that they support. Both immunogold (Furness et al., 2002) and immunofluorescence labeling for GLAST are greater in apical Deiters' cells, where OHC afferent innervation density is greater, than in basal Deiters' cells. Thus the gradient in afferent innervation density and GLAST in the $\mathrm{OHC}$ region are in a direction opposite to that seen with the IHCs; the two hair-cell systems therefore demonstrate independently a correspondence between GLAST expression and afferent innervation density. This suggests that there are regulatory mechanisms that match GLAST expression to the local requirement for glutamate uptake. Because VGLUT1 did not show similar gradients along the cochlea, it does not appear to be modulated by the same factors as GLAST.

With respect to the activity level of synapses, the most obvious relationship is demonstrated by high GLAST expression associated with the IHCs that have active synapses compared with low expression associated with the $\mathrm{OHCs}$ that have relatively inactive synapses; however, there is also a relationship between GLAST and fibers of different SRs around the IHC. In the cat, these fibers are spatially separated in the osseous spiral lamina, and their afferent endings are segregated around the IHC base. High-SR fibers are larger and terminate more on the pillar-cell side, whereas medium- and low-SR fibers are smaller and terminate on the modiolar side (Liberman, 1982). There appear to be similar separations at the osseous spiral lamina in guinea pig and gerbil (Tsuji and Liberman, 1997; Slepecky et al., 2000), but it has yet to be confirmed whether fibers of different SRs are segregated at the IHC like those observed in cats.

Our data indicate that the density of GLAST labeling is stronger on the pillar-cell side, corresponding with the likely location of high-SR fibers. Although the physiological basis of the different SRs is also not known, one possible factor is the amount of spontaneous glutamate release at the synapse. Thus, GLAST may be upregulated here because of normally higher levels of extracellular glutamate. Another possibility is that the high-SR fibers may be more sensitive and thus more susceptible to excitotoxicity. Thus GLAST may be needed in higher quantities around high-SR terminals to assist in ending synaptic action quickly and to provide more protection.

A number of studies on different systems provide evidence that glutamatergic activity affects transporter expression. Deafferentation of corticostriatal glutamatergic fibers results in downregulation of transporters (Levy et al., 1995). Application of glutamate and CAMP and activation of ionotropic receptors increases GLAST expression in astrocytes in vitro (Gegelashvili et al., 1996). Activation of different classes of metabotropic glutamate receptors (mGluRs) can also regulate astroglial GLAST expression. Group I agonists cause downregulation of GLAST, whereas group II agonists produce upregulation, probably via mGluR5 and mGluR3, respectively (Gegelashvili et al., 2000). Thus transporter expression may be regulated by local glutamate release.

The molecular machinery involved in this process in the cochlea could also include receptor proteins. Activation of both group I and II mGluRs affects IHC neurotransmission (Kleinlogel et al., 1999; Oestreicher et al., 2002). There is no evidence, however, that either ionotropic or metabotropic GluRs are present in the supporting cells that also express GLAST. It remains to be determined whether these receptors are involved in regulating GLAST expression in the cochlea. Among the ionotropic receptors, GluR4 receptor subunits appear to be present on both the presynaptic and postsynaptic membranes at the IHC synapse (Ottersen et al., 1998). The presynaptic GluR4 could act as autoreceptors, helping to detect levels of glutamate and modulating its release, which might secondarily affect GLAST expression.

\section{Conclusions}

Our data suggest that (1) GLAST expression in supporting cells corresponds with changes in afferent innervation density to both IHCs and OHCs and (2) the local distribution of GLAST also corresponds with the difference in synaptic activity between IHC and $\mathrm{OHC}$ and between fibers around individual IHCs. These findings are consistent with the hypothesis that GLAST is regu- 
lated by factors relating to the density and activity of glutamatergic innervation and to the amounts of glutamate released in the cochlea.

\section{References}

Bai L, Xu H, Collins JF, Ghishan FK (2001) Molecular and functional analysis of a novel neuronal vesicular glutamate transporter. J Biol Chem 276:36764-36769.

Bellochio EE, Reimer RJ, Fremau Jr RT, Edwards RH (2000) Uptake of glutamate into synaptic vesicles by an inorganic phosphate transporter. Science 289:847-849.

Cheatham MA, Zheng J, Long K (2001) VGLUT1 is present in cochlear hair cells. Assoc Res Otolaryngol Abstr 21:378.

Counter SA, Canelon B, Borg E, Aldskogius H (1997) Pattern of synaptophysin immunoreactivity in the efferent nerve terminals of the guinea pig cochlea. Neurosci Lett 222:199-203.

Danbolt NC (2001) Glutamate uptake. Prog Neurobiol 65:1-105.

Davies C, Tingley D, Kachar B, Wenthold RJ, Petralia RS (2001) Distribution of members of the PSD-95 family of MAGUK proteins at the synaptic region of inner and outer hair cells of the guinea pig cochlea. Synapse 40:258-268.

Furness DN, Lehre KP (1997) Immunocytochemical localisation of the high affinity glutamate/aspartate transporter, GLAST, in the rat and guineapig cochlea. Eur J Neurosci 9:1961-1969.

Furness DN, Hulme JA, Lawton DM, Hackney CM (2002) Distribution of the glutamate/aspartate transporter GLAST in relation to the afferent synapses of outer hair cells in the guinea pig cochlea. J Assoc Res Otolaryngol 3:234-247.

Gegelashvili G, Civenni G, Racagni G, Danbolt NC, Schousboe I, Schousboe A (1996) Glutamate receptor agonists up-regulate glutamate transporter GLAST in astrocytes. NeuroReport 8:261-265.

Gegelashvili G, Dehnes Y, Danbolt NC, Schousboe A (2000) The highaffinity glutamate transporters GLT1, GLAST, and EAAT4 are regulated via different signaling mechanisms. Neurochem Int 37:163-170.

Hakuba N, Koga K, Gyo K, Usami S, Tanaka K (2000) Exacerbation of noise-induced hearing loss in mice lacking the glutamate transporter GLAST. J Neurosci 20:8750-8753.

Hashimoto S, Kimura RS (1988) Computer-aided three-dimensional reconstruction of morphometry of the outer hair cells of the guinea pig cochlea. Acta Otolaryngol (Stockh) 105:64-74.

Hashimoto S, Kimura RS, Takasaka T (1990) Computer-aided threedimensional reconstruction of the inner hair cells and their nerve endings in the guinea pig cochlea. Acta Otolaryngol (Stockh) 109:228-234.

Jiang D, Furness DN, Hackney CM, Lopez DE (1993) Microslicing of the resin-embedded cochlea in comparison with the surface preparation technique for analysis of hair-cell number and morphology. Br J Audiol 27:195-203.

Kleinlogel S, Oestreicher E, Arnold T, Ehrenberger K, Felix D (1999) Metabotropic glutamate receptors group I are involved in cochlear neurotransmission. NeuroReport 10:1879-1882.

Lehre KP, Danbolt NC (1998) The number of glutamate transporter subtypes at glutamatergic synapses: chemical and stereological quantification in young adult rat brain. J Neurosci 18:8751-8757.

Lehre KP, Levy LM, Ottersen OP, Storm-Mathisen J, Danbolt NC (1995) Differential expression of two glial glutamate transporters in the rat brain: quantitative and immunocytochemical observations. J Neurosci 15:1835-1853.

Levy LM, Lehre KP, Walaas SI, Storm-Mathisen J, Danbolt NC (1995) Down-regulation of glial glutamate transporters after glutamatergic denervation in the rat brain. Eur J Neurosci 7:2036-2041.

Liberman MC (1982) Single-neuron labeling in the cat auditory nerve. Science 216:1239-1241.

Liberman MC, Dodds LW, Pierce S (1990) Afferent and efferent innervation of the cat cochlea: quantitative analysis with light and electron microscopy. J Comp Neurol 301:443-460.

Matsubara A, Laake JH, Davanger S, Usami S, Ottersen OP (1996) Organization of AMPA receptor subunits at a glutamate synapse: a quantitative
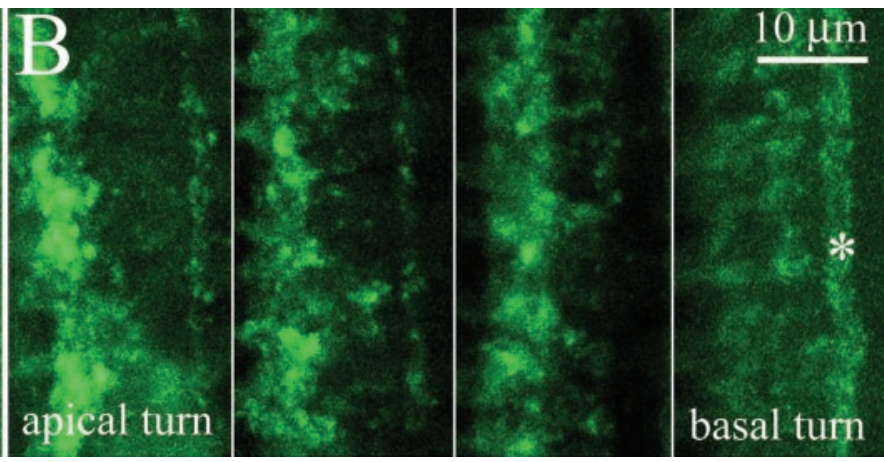

Figure 9. A, A single confocal image of IHCs labeled for VGLUT1. The IHCs can be seen by virtue of the faint fluorescence aes of the IHC region along the cochlear spiral after labeling for VGLUT1. Note that as well as labeling apparently IHC bases, there is also labeling in the inner spiral bundle $(*)$. No consistent qualitative difference in labeling regions of the cochlea.

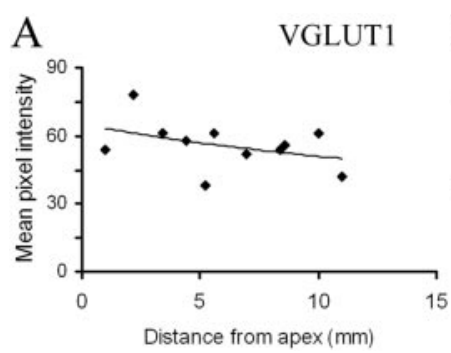

Figure 10. $A$, Graph showing fluorescent intensity in the IHC region as a function of distance from the apex along the cochlea after immunofluorescent labeling for VGLUT1. There is no evidence of a peak in labeling equivalent to that seen for GLAST in this sample. $B$, Graph showing fluorescent intensity for tubulin labeling from one animal. Note the absence of a significant peak in the labeling in the middle region. Second-order polynomial trend lines have been added to both to show the overall patterns of the data points.

immunogold analysis of hair cell synapses in the rat organ of Corti. J Neurosci 16:4457-4467.

Morrison D, Schindler RA, Wërsall J (1975) A quantitative analysis of the afferent innervation of the organ of Corti in guinea pig. Acta Otolaryngol (Stockh) 79:11-23.

Oestreicher E, Wolfgang A, Felix D (2002) Neurotransmission of the cochlear inner hair cell synapse-implications for inner ear therapy. Adv Otorhinolaryngol 59:131-139.

Ottersen OP, Takumi Y, Matsubara A, Landsend AS, Laake JH, Usami S (1998) Molecular organization of a type of peripheral glutamate synapse: the afferent synapses of hair cells in the inner ear. Prog Neurobiol 54:127-148.

Parks TN (2000) The AMPA receptors of auditory neurons. Hear Res 147:77-91.

Rebillard G, Ruel J, Nouvian R, Saleh H, Pujol R, Dehnes Y, Raymond J, Puel JL, Devau G (2003) Glutamate transporters in the guinea-pig cochlea: partial mRNA sequences, cellular expression and functional implications. Eur J Neurosci 17:83-92.

Slepecky NB, Galsky MD, Swartzentruber-Martin H, Savage J (2000) Study of afferent nerve terminals and fibers in the gerbil cochlea: distribution by size. Hear Res 144:124-134.

Spoendlin H (1972) Innervation densities of the cochlea. Acta Otolaryngol (Stockh) 73:235-248.

Storck T, Schulte S, Hofmann K, Stoffel W (1992) Structure, expression, and functional analysis of $\mathrm{Na}^{+}$-dependent glutamate/aspartate transporter from rat brain. Proc Natl Acad Sci USA 89:10955-10959.

Takumi Y, Matsubara A, Laake JH, Ramirez-Leon V, Roberg B, Torgner I, Kvamme E, Usami S, Ottersen OP (1999) Phosphate activated glutaminase is concentrated in mitochondria of sensory hair cells in rat inner ear: a high resolution immunogold study. J Neurocytol 28:223-237.

Tsuji J, Liberman MC (1997) Intracellular labeling of auditory nerve fibers in guinea pig: central and peripheral projections. J Comp Neurol 381:188-202. 